\title{
Understanding the origin of the extended $\gamma$-ray emission and the physical nature of HESS J1841-055 using observations at TeV energies with the MAGIC telescopes
}

\author{
Lab Saha, ${ }^{a, *}$ Alicia López-Oramas ${ }^{b}$ and David Green ${ }^{c, *}$ on behalf of the MAGIC \\ Collaboration \\ (a complete list of authors can be found at the end of the proceedings) \\ ${ }^{a}$ IPARCOS Institute and EMFTEL Department, Universidad Complutense de Madrid, E-28040 Madrid, \\ Spain \\ ${ }^{b}$ Inst. de Astrofísica de Canarias, E-38200 La Laguna, and Universidad de La Laguna, Dpto. Astrofísica, \\ E-38206 La Laguna, Tenerife, Spain \\ ${ }^{c}$ Max-Planck-Institut für Physik, D-80805 München, Germany \\ E-mail: labsaha@ucm.es, alicia.lopez@iac.es
}

With the improved sensitivity with respect to the previous generation, current space-borne and ground-based gamma-ray telescopes have made the number of $\gamma$-ray sources detected at GeV$\mathrm{TeV}$ energies increase many folds over the last decade. Many of the detected extended gamma-ray sources are not associated with any known sources at other wavelengths. Understanding the nature of these sources and the origin of the observed high energy gamma-ray emission remains a great challenge. Using the MAGIC telescopes, we have observed one such unassociated gamma-ray source, named HESS J1841-055 at TeV energies. We investigate the physical nature and origin of the $\gamma$-ray emission from this extended source. In this paper, we present the results of our detailed investigation on this source using MAGIC data and other multi-waveband information on nearby sources. The results included in this proceeding and shown in this presentation were published in ref. [1]. The details about the analysis, interpretation and modelling can be found in the aforementioned reference.

$37^{\text {th }}$ International Cosmic Ray Conference (ICRC 2021)

July 12 th - 23rd, 2021

Online - Berlin, Germany

\footnotetext{
${ }^{*}$ Presenter
} 
The unidentified $\gamma$-ray source HESS J1841-055 was first discovered at TeV energies in 2007 with a statistical significance of $10.7 \sigma$ by the High Energy Stereoscopic System (H.E.S.S.) during the Galactic plane survey [2]. The observed emission was reported as extended with an elliptical extension of $0.41^{\circ}$ and $0.25^{\circ}$ along the semi-major and semi-minor axes, respectively and centered at Right Ascension (RA): $18^{h} 40^{m} 55^{s}$ and Declination (Dec): $5^{\circ} 33^{\prime} 00^{\prime \prime}$ with a position angle $39^{\circ}$ relative to the RA axis. HESS J1841-055 is further observed with other experiments like ARGO-YBJ and HAWC and the observed $\gamma$-ray emission was also found to be extended [3, 4] .

This region was further investigated at other wavelengths to search for possible counterparts. Although no confirmed counterparts of the TeV source HESS J1841-055 at lower energies are known, several possible associations have been suggested. The detection of two separate extended sources (FGES J1839.4-0554 and FGES J1841.4-0514) was also reported in this region at energies above $10 \mathrm{GeV}$ using data from the Fermi-Large Area Telescope [LAT, 5, 6].

We have observed HESS J1841-055 between April 2012 and August 2013 using MAGIC telescopes, for a total of about 43 hours, at zenith angles between $5^{\circ}$ and $50^{\circ}$, resulting in an energy threshold for this analysis of $\sim 150 \mathrm{GeV}$. After quality cuts, which account for hardware problems, unusual rates, and bad atmospheric conditions, $\sim 34$ hours of high-quality, dark-time data are selected for further analysis. The analysis of the MAGIC data is performed using the standard MAGIC Analysis and Reconstruction Software [Mars; 7, 8] and standard analysis procedure. Given the extension of the source and the possibility of contamination from other nearby sources, we study the region using an iterative maximum likelihood method included in the Skyprism package [9]. Skyprism has specifically been developed to perform 2D fitting of Imaging Atmospheric Cherenkov Telescopes (IACTs) data and has been optimised for MAGIC data.

We also explore the $\mathrm{GeV}$ counterpart making use of 10-year data of Fermi-LAT. We finally model the GeV-TeV emission to unveil the dominant $\gamma$-ray emission mechanisms at work. The potential counterparts at other frequencies are also investigated. The low energy threshold of MAGIC, which allows to overlap with Fermi-LAT in the GeV domain, combined with the MAGIC capabilities of reaching several $\mathrm{TeV}$, make the MAGIC telescopes a suitable instrument to study this region within a broad energy range. The combination of both MAGIC and Fermi-LAT allows spectral studies of this complex region for almost four decades in energy.

In order to study the energy-dependent morphology of the extended source HESS J1841-055, we consider three different energy ranges: $50-500 \mathrm{GeV}$ (low energy, LE), $500 \mathrm{GeV}-1 \mathrm{TeV}$ (medium energy, ME) and $>1 \mathrm{TeV}$ (high energy, HE), respectively. We find that the extension of the source at these three energy ranges appears to be the same (approximately $0.4^{\circ}$ ). However, the overall detection significance of the extended emission reduces at higher energies, revealing only a few hotspots in the southern part of the source (see left panel of Fig. 1). MAGIC observations show that the source has an extension compatible with that measured by the H.E.S.S. collaboration at $\mathrm{TeV}$ energies. It is also evident from the different maps that the extended region shows several bright hotspots with a significance of more than $5 \sigma$. Many bright highly-significant spots are detected at LE and ME, while they mostly disappear at HE (see Fig. 1 of ref. [1]). These hotspots hint the presence of multiple sources in the region. It also indicates that the most significant emission at higher energies is coming from the southern part of the region.

As discussed above, the extended source HESS J1841-055 may potentially consist of multiple sources. To check this, we consider three different source models covering the full energy range, 

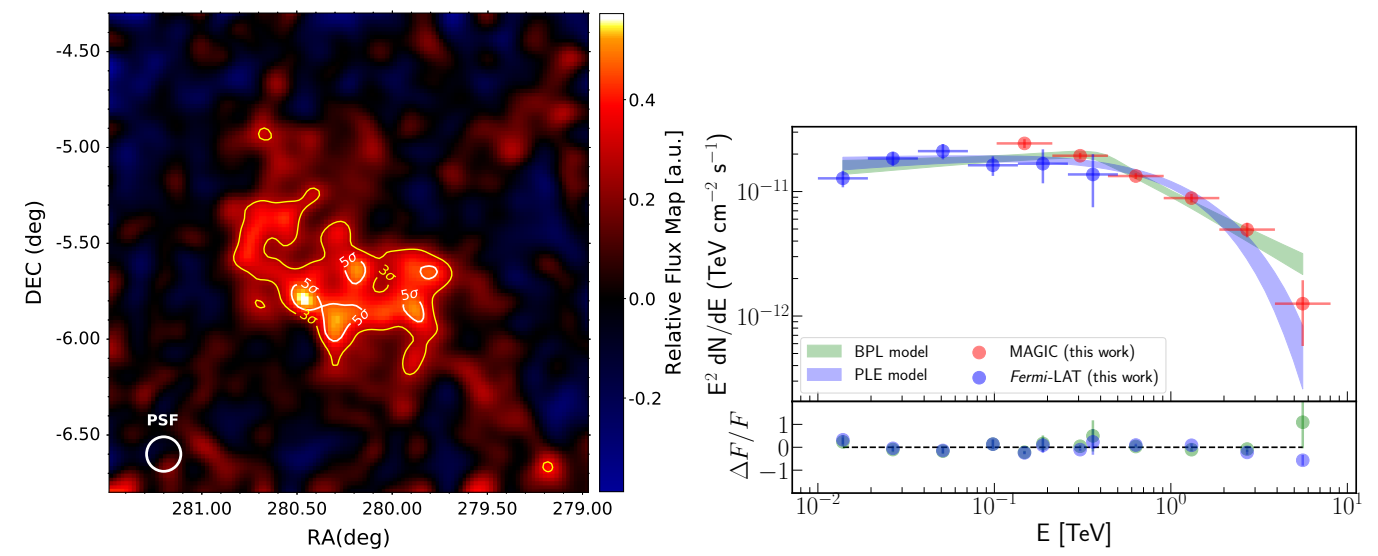

Figure 1: Left panel: $\gamma$-ray relative flux maps with $3 \sigma$ (yellow) and $5 \sigma$ (white) contour levels of the extended source HESS J1841-055 as seen by MAGIC is for HE ( $>1 \mathrm{TeV})$. Right panel: SED of both MAGIC and Fermi-LAT observations.

i.e., energies from $50 \mathrm{GeV}$ to above $1 \mathrm{TeV}$. We first consider a single-source model where the extended source is considered to be a $2 \mathrm{D}$ elliptical Gaussian. We leave the peak position, extension along $\mathrm{X}$ and $\mathrm{Y}$ direction and angle w.r.t. the $\mathrm{X}$ direction free while maximizing the likelihood value of the fit. For the second model, we replace the single-source model with two sources which are modelled as $2 \mathrm{D}$ circular Gaussian. The peak position and radius ( $\sigma \sigma$ standard deviation) of the two sources are free parameters of the model. Finally, for the third option, we model the entire source region considering three different sources, one with elliptical disk model and the other two with Gaussian models. It is found that both two-source model and three-source models are better than a single-source model. The improvement of the two-source model w.r.t. to the one-source model is given by TS $=9.4$ for 1 additional degree of freedom (d.o.f.), which corresponds to an improvement at $3 \sigma$. The improvement of the three-source model w.r.t. to the one-source model is given by test-statistics of 16.2 for additional 5 d.o.f which corresponds to an improvement of $2.7 \sigma$. This hints that the HESS J1841-055 region is better modelled by multiple sources.

The spectral energy distribution (SED) is calculated in the energy range of $50 \mathrm{GeV}$ to $>1 \mathrm{TeV}$, using the Skyprism package. We consider the extended 2D Gaussian template with the extension $0.4^{\circ}$ at the position of the HESS J1841-055 and an isotropic background. The observed spectrum in this energy range is best described by a power-law model with spectral index of $2.57 \pm 0.05$. We have also combined both MAGIC and Fermi-LAT SED points and performed a joint likelihood fit. Both the broken power-law and power-law with exponential cutoff models describe the combined SED better than a simple power-law model, implying that a significant curvature is present in the SED (see right panel of Fig 1).

Multi-wavelength modelling of the data indicates that the leptonic model can explain the data well. Due to the higher ambient matter density, the bremsstrahlung spectrum dominates over IC spectrum. The radio and X-ray fluxes put a constraint on the magnetic field of $5 \mu \mathrm{G}$ in the emission volume when they are accounted with a synchrotron emission process. The magnetic field of $5 \mu \mathrm{G}$ is very close to that of some other known old pulsar wind nebulae (PWNe) [10, 11]. Given the high ambient matter density and presence of molecular clouds, a hadronic emission model is also 
suitable to explain the observed data at $\mathrm{GeV}-\mathrm{TeV}$ energies. We find that the hadronic model can explain the data very well for a BPL proton distribution and an ambient matter density of $100 \mathrm{~cm}^{-3}$.

In the whole discussion on multi-wavelength modelling of the data, our assumption was that the observed emission is entirely due to a single source. However, we have already seen that the region is populated by different sources which were established through observations at lower energy bands. Some of them could be potentially associated with the observed emission at GeV-TeV energies following their location and energetics. Given the angular resolution of the $\gamma$-ray telescopes at present generation, it is not possible to have an unambiguous association with the sources at other wavebands. One possible scenario for the extension of the emission is the interaction of runaway cosmic particles from the source and the $\gamma$-ray visibility is enhanced due to interaction with molecular clouds which are covering the extended source very well (see Fig. 8 of [1]). The presence of molecular clouds along the extension of the source also supports the relatively high ambient matter density required for both leptonic and hadronic model.

The nature of this source is ambiguous due to its extension at very high energies and presence of several different class of the objects. The observations at X-ray energies did not show any bright synchrotron nebula around the pulsars present in this region. However, in this scenario the absence of bright synchrotron nebula can be easily explained. If the $\mathrm{TeV}$ source is powered by one or several pulsars present in this region, then pulsars are expected to be relic ones. For such PWNe, IC emission efficiency is more pronounced due to lower magnetic fields. In our multi-waveband modelling, we find that the IC contribution for this source is $10 \%$ less compared to the bremsstrahlung spectrum. Therefore, it is reasonable to consider that the synchrotron emission could be even more inefficient, which supports the absence of the bright synchrotron nebula around the pulsars. Therefore, if the bright $\mathrm{TeV}$ emission is assumed to be associated with a PWN, then the PWN requires to be a relic one where the remnant of the supernova explosion has already disappeared. We therefore conclude that the observed emission can be potentially most likely associated with a PWN. In an SNR scenario, a strong association can be made provided that the observed emission is considered due to the particles that escaped the SNR shocks and are interacting with the molecular clouds. Following the diffusion timescale $\left(t_{\text {diff }}=17 \mathrm{kyr}\right.$ ) and the age of the SNR G26.6-0.1 as a middle-age SNR [ $\sim 10^{3}$ years; 13], it can be considered a possible candidate for at least part of the detected $\mathrm{GeV}-\mathrm{TeV}$ emission.

In summary, our deep study of the unidentified extended $\gamma$-ray source HESS J1841-055 at TeV energies shows that the observed $\gamma$-ray emission from HESS J1841-055 is significantly extended and is consistent with other measurements. Within the present morphological and spectral studies of this extended source using $\mathrm{GeV}-\mathrm{TeV}$ data and available MWL information on sources present within the region, we find that the extended $\gamma$-ray emission seems to be associated with multiple sources in this region. The observed SED can be explained well with both a leptonic (bremsstrahlung) and a hadronic model for the density of ambient matter of $100 \mathrm{~cm}^{-3}$ assuming a broken power-law distribution of electrons and protons, respectively. The GeV-TeV emission is compatible with a PWN scenario, although a fraction of the $\gamma$-ray emission can also be explained within a SNR scenario. However, disentangling these sources at $\mathrm{TeV}$ energies (either point sources or extended sources) one from another and quantifying their contribution to the observed morphology of the source demands much better angular resolution compared to the present generation of $\gamma$-ray telescopes. Hence, it becomes naturally an interesting source of study for the next generation of IACT telescopes. 
Acknowledgement: We acknowledge the support from the agencies and organizations listed here: https://magic.mpp.mpg.de/acknowledgments_ICRC2021

\section{References}

[1] MAGIC Collaboration, Acciari, V. A., Ansoldi, S., et al. 2020, MNRAS, 497, 3734. doi:10.1093/mnras/staa2135

[2] Aharonian F., Akhperjanian A. G., Barres de Almeida U., Bazer-Bachi A. R., Behera B., others 2008, A\&A, 477, 353

[3] Bartoli B., et al., 2013, ApJ, 767, 99

[4] Abeysekara A. U., et al., 2017, ApJ, 843, 40

[5] Ackermann M., Ajello M., Baldini L., Ballet J., Barbiellini G., Bastieri D., Bellazzini R., others 2017, ApJ, 843, 139

[6] Ajello M., et al., 2017, ApJS, 232, 18

[7] Moralejo A., et al., 2009, preprint, (arXiv:0907.0943)

[8] Zanin R., Carmona E., J. S., Colin P., Frantzen K., Gaug M., others 2013, preprint, (arXiv:0907.0943)

[9] Vovk I., Strzys M., Fruck C., 2018, A\&A, 619, A7

[10] Kargaltsev O., Rangelov B., Pavlov G. G., 2013, preprint, (arXiv: 1305.2552)

[11] Reynolds S. P., Gaensler B. M., Bocchino F., 2012, SSR, 166, 231

[12] Aleksić J., et al., 2012, Astroparticle Physics, 35, 435

[13] Bamba A., Ueno M., Koyama K., Yamauchi S., 2003, ApJ, 589, 253 


\section{Full Authors List: MAGIC Collaboration}

V. A. Acciari ${ }^{15}$, S. Ansoldi ${ }^{38,50}$, L. A. Antonelli ${ }^{17}$, A. Arbet Engels ${ }^{10}$, M. Artero ${ }^{16}$, K. Asano $^{22}$, D. Baack ${ }^{9}$, A. Babić ${ }^{7}$, A. Baquero ${ }^{29}$, U. Barres de Almeida $^{1}$, J. A. Barrio ${ }^{29}$, I. Batković ${ }^{31}$, J. Becerra González ${ }^{15}$, W. Bednarek ${ }^{28}$, L. Bellizzi ${ }^{33}$, E. Bernardini ${ }^{8}$, M. Bernardos ${ }^{31}$, A. Berti ${ }^{30}$, J. Besenrieder ${ }^{30}$, W. Bhattacharyya ${ }^{8}$, C. Bigongiari ${ }^{17}$, A. Biland ${ }^{10}$, O. Blanch ${ }^{16}$, H. Bökenkamp ${ }^{9}$, G. Bonnoli $^{14}$, Ž. Bošnjak $^{7}$, G. Busetto ${ }^{31}$, R. Carosi ${ }^{32}$, G. Ceribella ${ }^{30}$, M. Cerruti ${ }^{37}$, Y. Chai $^{30}$, A. Chilingarian ${ }^{41}$, S. Cikota ${ }^{7}$, S. M. Colak ${ }^{16}$, E. Colombo ${ }^{15}$, J. L. Contreras ${ }^{29}$, J. Cortina ${ }^{2}$, S. Covino ${ }^{17}$, G. D’Amico ${ }^{30,48}$, V. D’Elia ${ }^{17}$, P. Da Vela ${ }^{32,43}$, F. Dazzi ${ }^{17}$, A. De Angelis ${ }^{31}$, B. De Lotto ${ }^{38}$, M. Delfino ${ }^{16,49}$, J. Delgado ${ }^{16,49}$, C. Delgado Mendez $^{2}$, D. Depaoli ${ }^{18}$, F. Di Pierro ${ }^{18}$, L. Di Venere ${ }^{19}$, E. Do Souto Espiñeira ${ }^{16}$, D. ${\text { Dominis } \text { Prester }^{6} \text {, A. Donini }}^{38}$, D. Dorner ${ }^{39}$, M. Doro ${ }^{31}$, D. Elsaesser ${ }^{9}$, V. Fallah Ramazani ${ }^{11,44}$, A. Fattorini ${ }^{9}$, M. V. Fonseca ${ }^{29}$, L. Font $^{36}$, C. Fruck ${ }^{30}$, S. Fukami ${ }^{22}$, Y. Fukazawa ${ }^{25}$, R. J. García López ${ }^{15}$, M. Garczarczyk ${ }^{8}$, S. Gasparyan ${ }^{40}$, M. Gaug ${ }^{36}$, N. Giglietto ${ }^{19}$, F. Giordano ${ }^{19}$, P. Gliwny ${ }^{28}$, N. Godinović ${ }^{4}$, D. Green ${ }^{30}$, J. G. Green ${ }^{17}$, D. Hadasch ${ }^{22}$, A. Hahn ${ }^{30}$, L. Heckmann ${ }^{30}$, J. Herrera ${ }^{15}$, J. Hoang ${ }^{29,45}$, D. Hrupec ${ }^{5}$, M. Hütten ${ }^{30}$, T. Inada ${ }^{22}$, K. Ishio ${ }^{28}$, Y. Iwamura ${ }^{22}$, I. Jiménez Martínez ${ }^{2}$, J. Jormanainen ${ }^{11}$, L. Jouvin ${ }^{16}$, M. Karjalainen ${ }^{15}$, D. Kerszberg ${ }^{16}$, Y. Kobayashi ${ }^{22}$, H. Kubo ${ }^{23}$, J. Kushida ${ }^{24}$, A. Lamastra ${ }^{17}$, D. Lelas ${ }^{4}$, F. Leone ${ }^{17}$, E. Lindfors ${ }^{11}$, L. Linhoff $^{9}$, S. Lombardi ${ }^{17}$, F. Longo ${ }^{38,46}$, R. López-Coto ${ }^{31}$, M. López-Moya ${ }^{29}$, A. López-Oramas ${ }^{15}$, S. Loporchio ${ }^{19}$, B. Machado de Oliveira Fraga $^{1}$, C. Maggio ${ }^{36}$, P. Majumdar ${ }^{34}$, M. Makariev ${ }^{35}$, M. Mallamaci ${ }^{31}$, G. Maneva ${ }^{35}$, M. Manganaro ${ }^{6}$, K. Mannheim ${ }^{39}$, L.

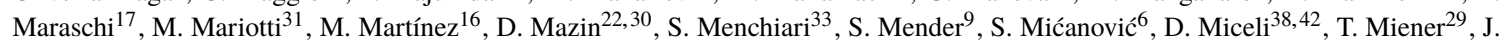
M. Miranda ${ }^{33}$, R. Mirzoyan ${ }^{30}$, E. Molina ${ }^{37}$, A. Moralejo ${ }^{16}$, D. Morcuende ${ }^{29}$, V. Moreno ${ }^{36}$, E. Moretti ${ }^{16}$, T. Nakamori ${ }^{26}$, L. Nava ${ }^{17}$, V. Neustroev $^{12}$, C. Nigro ${ }^{16}$, K. Nilsson ${ }^{11}$, K. Nishijima ${ }^{24}$, K. Noda $^{22}$, S. Nozaki ${ }^{23}$, Y. Ohtani ${ }^{22}$, T. Oka ${ }^{23}$, J. Otero-Santos ${ }^{15}$, S. Paiano ${ }^{17}$, M. Palatiello ${ }^{38}$, D. Paneque ${ }^{30}$, R. Paoletti ${ }^{33}$, J. M. Paredes ${ }^{37}$, L. Pavletić ${ }^{6}$, P. Peñil ${ }^{29}$, M. Persic ${ }^{38,17}$, M. Pihet ${ }^{30}$, P. G. Prada Moroni ${ }^{32}$, E. Prandini ${ }^{31}$, C. Priyadarshi ${ }^{16}$, I. Puljak ${ }^{4}$, W. Rhode ${ }^{9}$, M. Ribó ${ }^{37}$, J. Rico ${ }^{16}$, C. Righi ${ }^{17}$, A. Rugliancich ${ }^{32}$, N. Sahakyan ${ }^{40}$, T. Saito ${ }^{22}$, S. Sakurai $^{22}$, K. Satalecka ${ }^{8}$, F. G. Saturni ${ }^{17}$, B. Schleicher ${ }^{39}$, K. Schmidt $^{9}$, T. Schweizer ${ }^{30}$, J. Sitarek ${ }^{28}$, I. Šnidarić ${ }^{3}$, D. Sobczynska ${ }^{28}$, A. Spolon $^{31}$, A. Stamerra ${ }^{17}$, J. Strišković ${ }^{5}$, D. Strom ${ }^{30}$, M. Strzys ${ }^{22}$, Y. Suda ${ }^{25}$, T. Surić ${ }^{3}$, M. Takahashi ${ }^{22}$, R. Takeishi ${ }^{22}$, F. Tavecchio ${ }^{17}$, P. Temnikov ${ }^{35}$, T. Terzicic ${ }^{6}$, M. Teshima ${ }^{30,22}$, L. Tosti ${ }^{20}$, S. Truzzi ${ }^{33}$, A. Tutone ${ }^{17}$, S. Ubach ${ }^{36}$, J. van Scherpenberg ${ }^{30}$, G. Vanzo $^{15}$, M. $^{3}$. Vazquez Acosta $^{15}$, S. Ventura ${ }^{33}$, V. Verguilov ${ }^{35}$, C. F. Vigorito ${ }^{18}$, V. Vitale ${ }^{21}$, I. Vovk ${ }^{22}$, M. Will ${ }^{30}$, C. Wunderlich ${ }^{33}$, T. Yamamoto ${ }^{27}$, D. Zarić ${ }^{4}$, and L. Saha ${ }^{29}$

${ }^{1}$ Centro Brasileiro de Pesquisas Físicas (CBPF). ${ }^{2}$ Centro de Investigaciones Energéticas, Medioambientales y Tecnológicas (CIEMAT). ${ }^{3}$ Croatian MAGIC Group: Ruđer Bošković Institute. ${ }^{4}$ Croatian MAGIC Group: University of Split, Faculty of Electrical Engineering, Mechanical Engineering and Naval Architecture (FESB). ${ }^{5}$ Croatian MAGIC Group: Josip Juraj Strossmayer University of Osijek, Department of Physics. ${ }^{6}$ Croatian MAGIC Group: University of Rijeka, Department of Physics. ${ }^{7}$ Croatian MAGIC Group: University of Zagreb, Faculty of Electrical Engineering and Computing (FER). ${ }^{8}$ Deutsches Elektronen-Synchrotron (DESY). ${ }^{9}$ Technische Universität Dortmund. ${ }^{10}$ ETH Zürich. ${ }^{11}$ Finnish MAGIC Group: Finnish Centre for Astronomy with ESO, University of Turku. ${ }^{12}$ Finnish MAGIC Group: Astronomy Research Unit, University of Oulu. ${ }^{13}$ Department of Astronomy, University of Geneva. ${ }^{14}$ Instituto de Astrofísica de Andalucía-CSIC. ${ }^{15}$ Instituto de Astrofísica de Canarias and Dpto. de Astrofísica, Universidad de La Laguna. ${ }^{16}$ Institut de Física d'Altes Energies (IFAE), The Barcelona Institute of Science and Technology (BIST). ${ }^{17}$ National Institute for Astrophysics (INAF). ${ }^{18}$ INFN MAGIC Group: INFN Sezione di Torino and Università degli Studi di Torino. ${ }^{19}$ INFN MAGIC Group: INFN Sezione di Bari and Dipartimento Interateneo di Fisica dell'Università e del Politecnico di Bari. ${ }^{20}$ INFN MAGIC Group: INFN Sezione di Perugia. ${ }^{21}$ INFN MAGIC Group: INFN Roma Tor Vergata. ${ }^{22}$ Japanese MAGIC Group: Institute for Cosmic Ray Research (ICRR), The University of Tokyo. ${ }^{23}$ Japanese MAGIC Group: Department of Physics, Kyoto University. ${ }^{24}$ Japanese MAGIC Group: Department of Physics, Tokai University. ${ }^{25}$ Japanese MAGIC Group: Physics Program, Graduate School of Advanced Science and Engineering, Hiroshima University. ${ }^{26}$ Japanese MAGIC Group: Department of Physics, Yamagata University. ${ }^{27}$ Japanese MAGIC Group: Department of Physics, Konan University. ${ }^{28}$ University of Lodz, Faculty of Physics and Applied Informatics, Department of Astrophysics. ${ }^{29}$ IPARCOS Institute and EMFTEL Department, Universidad Complutense de Madrid. ${ }^{30}$ Max-Planck-Institut für Physik. ${ }^{31}$ Università di Padova and INFN. ${ }^{32}$ Università di Pisa and INFN Pisa. ${ }^{33}$ Università di Siena and INFN Pisa. ${ }^{34}$ Saha Institute of Nuclear Physics. ${ }^{35}$ Inst. for Nucl. Research and Nucl. Energy, Bulgarian Academy of Sciences. ${ }^{36}$ Departament de Física, and CERES-IEEC, Universitat Autónoma de Barcelona. ${ }^{37}$ Universitat de Barcelona, ICCUB, IEEC-UB. ${ }^{38}$ Università di Udine and INFN Trieste. ${ }^{39}$ Universität Würzburg. ${ }^{40}$ Armenian MAGIC Group: A. Alikhanyan National Science Laboratory. ${ }^{41}$ Armenian MAGIC Group: ICRANet-Armenia at NAS RA. ${ }^{42}$ Laboratoire d'Annecy de Physique des Particules (LAPP), CNRS-IN2P3. ${ }^{43}$ University of Innsbruck. ${ }^{44}$ Ruhr-Universität Bochum, Fakultät für Physik und Astronomie, Astronomisches Institut (AIRUB). ${ }^{45}$ Department of Astronomy, University of California Berkeley. ${ }^{46}$ Dipartimento di Fisica, Università di Trieste. ${ }^{47}$ Dept. of Physics and Astronomy, University of Bologna. ${ }^{48}$ Department for Physics and Technology, University of Bergen. ${ }^{49}$ Port d'Informació Científica (PIC). ${ }^{50}$ International Center for Relativistic Astrophysics (ICRA). 